\title{
Bibliography of Professor Dirven
}

Vries, D. M. de \& J. G. P. Dirven, 1948. Sociologisch en oecologisch onderzoek van grasland. Verslag CILO 1948: 11-14.

Hart, M. L. 't \& J. G. P. Dirven, 1949. De grasopbrengsten in 1949. Verslag CILO 1949: $54-57$.

Nielen, G. Chr. \& J. G. P. Dirven, 1949. The accuracy of the $25 \mathrm{~cm}^{2}$ specific frequency method. Stencil CILO, S. 662: 1-4.

Vries, D. M. de, Th. A. de Boer \& J. G. P. Dirven, 1949. Waardering van grasland en beoordeling van bodemeigenschappen op grond van de botanische samenstelling. Landbouwkundig Tijdschrift 61: 347-356.

Vries, D. M. de, Th. A. de Boer \& J. G. P. Dirven, 1949. Evaluation of grassland by botanical research in the Netherlands. United Nations Scientific Conference on the Conservation and Utilization of Resources (7 April 1949): 522-528.

Dirven, J. G. P., A. Bakker \& A. Kemp, 1950. Oriënterend onderzoek naar de samenstelling van de grasmat der boomgaarden in het rivierkleigebied (Project 3). Verslag CILO 1950: 26-30.

Dirven, J. G. P. \& A. Kemp, 1950. Oriënterend onderzoek naar de samenstelling van de grasmat der boomgaarden in het rivierkleigebied. Gestencilde Mededelingen CILO, No 15: 1-8.

Hart, M. L. 't \& J. G. P. Dirven, 1950. De grasopbrengsten in 1950. Verslag CILO 1950: 66-68.

Nielen, G. Chr. \& J. G. P. Dirven, 1950. De nauwkeurigheid van de plantensociologische $1 / 4 \mathrm{dm}^{2}$ frequentiemethode. Verslagen Landbouwkundige Onderzoekingen 56.13: 1-27.

Dirven, J. G. P. \& D. M. de Vries, 1951. Oecologisch graslandonderzoek. Nederlandsch Kruidkundig Archief 58: 10-11.

Dirven, J. G. P., 1952. Verslag van een studiereis naar de U.S.A. en het Caraibisch gebied. Stencil, Dept. LVV, Surinam, 34 pp.

Dirven, J. G. P. \& J. J. v.d. Eijk, 1952. Verslag van een studiereis naar Brits Guyana. Stencil, Dept. LVV, Surinam, $36 \mathrm{pp}$.

Dirven, J. G. P., 1953. De natuurlijke graslanden in Suriname. I. Plantkundige samenstelling. De Surinaamse Landbouw 1: 269-272.

Dirven, J. G. P., 1953. Meer en beter gras. Landbouwnieuws, Dept. LVV, Surinam, maart-april 1953: 6-12.

Dirven, J. G. P. \& J. A. H. Hendriks, 1953. Grasgroei op vier grondsoorten van het Lelydorpplan (1). De Surinaamse Landbouw 1: 66-77.

Dirven, J. G. P. \& J. A. H. Hendriks, 1953. Een voederproef met zebu's op het Lelydorpplan. De Surinaamse Landbouw 1: 114-120.

Dirven, J. G. P. \& A. G. Smit, 1953. Onkruiden in rijstvelden. De Surinaamse Landbouw 1: 14-19.

Dirven, J. G. P., 1954. De natuurlijke graslanden in Suriname. II. Het opbrengstniveau. De Surinaamse Landbouw 2: 13-16. 
Dirven, J. G. P., 1954. De natuurlijke graslanden in Suriname. III. De voederwaarde. De Surinaamse Landbouw 2: 105-109.

Dirven, J. G. P. \& J. A. H. Hendriks, 1954. Grasgroei op vier grondsoorten van het Lelydorpplan (2). De Surinaamse Landbouw 2: 137-146.

Dirven, J. G. P., J. A. H. Hendriks \& E. J. H. Glavimans, 1954. Groeicurven van rundvee op het Lelydorpplan. De Surinaamse Landbouw 2: 198-201.

Dirven, J. G. P., 1955. Verbetering van grasland met geïmporteerde grassoorten. De Surinaamse Landbouw 3: 30-37.

Dirven, J. G. P., J. A. H. Hendriks \& E. J. H. Glavimans, 1955. De onkruidvegetatie op gronden van de Oude Kustvlakte. De Surinaamse Landbouw 3: 199-208.

Dirven, J. G. P. \& H. Jonge Poerink, 1955. Weeds in rice and their control in Suriname. Tropical Agriculture 32: 115-123.

Dirven, J. G. P., J. A. H. Hendriks \& E. J. H. Glavimans, 1956. Graslandverbetering op het Lelydorpplan in 1955. De Surinaamse Landbouw 4: 113-118.

Appelman, H. \& J. G. P. Dirven, 1957. Paragras op de Landsboerderij. De Surinaamse Landbouw 5: 123-129.

Dirven, J. G. P., 1958. Betere weidegrassen. Landbouwnieuws, Dept. LVV, Surinam, No 6: 12-15.

Appelman, H. \& J. G. P. Dirven, 1959. Melkproductie op pangolagras. De Surinaamse Landbouw 7: 21-36.

Appelman, H., J. G. P. Dirven \& A. R. Thong A Hung, 1959. Melkproductie op enige particuliere bedrijven. De Surinaamse Landbouw 7: 129-135.

Dirven, J. G. P., 1959. Grasland en veevoedergewassen. Landbouwproefstation, Paramaribo, Mededeling 21: 59-69.

Dirven, J. G. P., 1959. Gras en grondsoort. Landbouwnieuws, Dept. LVV, Surinam, No 9: 99.

Dirven, J. G. P., 1960. Grasland en veevoedergewassen. Landbouwproefstation, Paramaribo, Mededeling 22: 78-85.

Dirven, J. G. P., 1960. Palissadegras, een uitstekend weidegras. Landbouwnieuws, Dept. LVV, Surinam, mei: 62-63.

Dirven, J. G. P., I. G. H. Dulder \& W. Ch. Hermelijn, 1960. De braakvegetaties op rijstvelden in Nickerie. De Surinaamse Landbouw 8: 1-7.

Dirven, J. G. P. \& V. K. R. Ehrencron, 1960. Gebreksverschijnselen bij Pueraria phaseoloides. Kali 45: 181-187.

Dirven, J. G. P. \& H. A. van Hoof, 1960. A destructive virus disease of pangolagrass. Tijdschrift over Plantenziekten 66: 344-349.

Dirven, J. G. P. \& V. K. R. Ehrencron, 1961. Gebreksverschijnselen bij Pueraria phaseoloides. De Surinaamse Landbouw 9: 41-47.

Dirven, J. G. P. \& V. K. R. Ehrencron, 1961. Signes de carence sur Pueraria phaseoloides. Fertilité (Revue trimestrielle) (Mars-Avril): 21-29.

Dirven, J. G. P. \& H. A. van Hoof, 1961. A destructive virus disease of pangolagrass. Journal of the Agricultural Society of Trinidad and Tobago 61: 317-326.

Appelman, H. \& J. G. P. Dirven, 1962. Zoutopname door slachtvee. De Surinaamse Landbouw 10: 27-29.

Appelman, H. \& J. G. P. Dirven, 1962. Slachtvee in Suriname. Landbouwproefsta- 


\section{BIBLIOGRAPHY OF PROFESSOR DIRVEN}

tion, Paramaribo, Mededeling 30; $56 \mathrm{pp}$.

Appelman, \& J. G. P. Dirven, 1962. De invloed van de maaitijd op de chemische samenstelling van verschillende grassoorten. De Surinaamse Landbouw 10: 95102.

Appelman, H., J. G. P. Dirven \& V. K. R. Ehrencron, 1962. De pH in urine van melkvee op de Landsboerderij. De Surinaamse Landbouw 10: 161-167.

Appelman, H., J. G. P. Dirven \& H. Kuil, 1962. Giftigheid van Grotalaria quinquefolia als veevoer. De Surinaamse Landbouw 10: 67-70.

Appelman, H., J. G. P. Dirven \& A. R. Tjong A Hung, 1962. Intensive small-scale dairy farming on a pilot farm in Suriname. De Surinaamse Landbouw 10: 59-66.

Appelman, H., J. G. P. Dirven \& A. R. Tjong A Hung, 1962. Opfok van jongvee. De Surinaamse Landbouw 10: 118-121.

Appelman, H., J. G. P. Dirven \& A. R. Tjong A Hung, 1962. De gehalten aan koper en mangaan in haar van rundvee. De Surinaamse Landbouw 10: 157-160.

Dirven, J. G. P., 1962. Grasland. Landbouwproefstation, Paramaribo, Mededeling 29; 33 pp.

Dirven, J. G. P., 1962. De voederwaarde van bladeren en stengels bij tropische grassen. De Surinaamse Landbouw 10: 199-202.

Dirven, J. G. P., 1962. Grasland en groenvoedergewassen. Landbouwproefstation, Paramaribo, Mededeling 31: 68-78.

Dirven, J. G. P., 1962. Chemical composition and nutritive value of tropical kudzu (Pueraria phaseoloides (Roxb.) Benth). Bulletin Guyana Congress 1962.

Dirven, J. G. P., 1962. The protein content in Surinam roughages. Bulletin Guyana Congress 1962.

Dirven, J. G. P., I. G. H. Dulder \& A. R. Tjong A Hung, 1962. De melkveeteelt op het Lelydorpplan. De Surinaamse Landbouw 10: 203-209.

Dirven, J. G. P. \& Ph. Samson, 1962. Verslag van het onderzoek van de toestand der weilanden en middelen tot verbetering door E. Essed Bsc. De Surinaamse Landbouw 10: 142-148.

Dijkstra, N. D. \& J. G. P. Dirven, 1962. Digestibility and feeding value of some tropical grasses and kudzu. Netherlands Journal of Agricultural Science 10: 275285.

Appelman, H., J. G. P. Dirven \& A. R. Tjong A Hung, 1963. Intensive small-scale dairy farming on a pilot farm in Suriname. Caribbean Agriculture 1: 85-94.

Dirven, J. G. P., 1963. Problemen bij de graslandverbetering in Suriname. 15e Bijeenkomst NVWV, 26 maart 1963.

Dirven, J. G. P., 1963. Snijmais in de tropen. De Surinaamse Landbouw 11: 31-34.

Dirven, J. G. P., 1963. The nutritive value of the indigenous grasses of Surinam. Netherlands Journal of Agricultural Science 11: 295-307.

Dirven, J. G. P. \& V. K. R. Ehrencron, 1963. Mineralen in koedzoe. De Surinaamse Landbouw 11: 10-18.

Dirven, J. G. P. \& V. K. R. Ehrencron, 1963. Bemestingsproef bij koedzoe (Pueraria phaseoloides (Roxb.) Benth). De Surinaamse Landbouw 11: 39-45.

Dirven, J. G. P. \& V. K. R. Ehrencron, 1963. Drogestofgehalten bij grassen in de humide tropen. De Surinaamse Landbouw 11: 88-93. 
Dirven, J. G. P. \& A. R. Tjong A Hung, 1963. Rupsenvraat bij grassen. De Surinaamse Landbouw 11: 66-70.

Dirven, J. G. P., 1964. Chemical composition and nutritive value of tropical kudzu (Pueraria phaseoloides (Roxb.) Benth). Qualitas Plantarum 236/1-13.

Dirven, J. G. P., 1964. The protein content in Surinam roughages. Qualitas Plantarum 237/1-13.

Dirven, J. G. P. \& V. K. R. Ehrencron, 1964. Sporenelementen in weidegronden. De Surinaamse Landbouw 12: 11-21.

Dirven, J. G. P., 1965. Verslag IXe Internationale Grasland Congres 1965, Sao Paulo, Brazilië. Gestencilde mededeling 5, Afd. Landbouwplantenteelt en Graslandcultuur: 1-38.

Dirven, J. G. P., 1965. Milk production on grassland in Surinam. Proceedings 9th International Grassland Congress (Sao Paulo, Brazil): 995-999.

Dirven, J. G. P., 1965. Some important grassland types in Surinam. Netherlands Journal of Agricultural Science 13: 102-113.

Dirven, J. G. P., 1965. Enige belangrijke graslandtypen in Suriname. De Surinaamse Landbouw 13: 1-16.

Dirven, J. G. P., 1966. Het IXe Internationale Grasland Congres te Sao Paulo, Brazilië. Gebundelde Verslagen NVWV 3: 10-12.

Dirven, J. G. P., 1966. Zaden in de 0-5 cm zodelaag van beschuitgrasland. De Surinaamse Landbouw 14: 90-95.

Deinum, B. \& J. G. P. Dirven, 1967. Een oriënterende proef omtrent de invloed van licht en temperatuur op de opbrengst en samenstelling van Brachiaria ruziziensis. De Surinaamse Landbouw 15: 5-10.

Dirven, J. G. P., 1967. Perspectief van het tropische grasland. Openbare les aanvaarding ambt van Lector, 4-19.

Vries, D. M. de \& J. G. P. Dirven, 1967. Welke samenstelling van de zode is voor blijvend grasland wenselijk? Kali 71: 3-9.

Dirven, J. G. P., 1968. De onkruidvegetatie in de akkerbouwgewassen op lemige zandgronden van de Oude Kustvlakte. De Surinaamse Landbouw 16: 123-130.

Dirven, J. G. P., 1969. Graslandoverdenkingen. Kali-berichten 2: 3-8.

Dirven, J. G. P., 1969. De huidige graslandexploitatie, eenzijdige grasbestanden en de monocultuur. Gebundelde verslagen NVWV 8: 16-20.

Dirven, J. G. P., B. J. Hoogers \& D. M. de Vries, 1969. Interrelation between frequency, dominance and dry-weight percentage of species in grassland vegetations. Netherlands Journal of Agricultural Science 17: 161-166.

Dirven, J. G. P., 1970. Die Aufgabe der Gräserzüchtung in den Tropen unter besonderer Berücksichtigung von Vermehrung, Ertrag und Futterwert. Zeitschrift für Kulturtechnik und Flurbereinigung 11: 92-107.

Dirven, J. G. P., 1970. De onkruidvegetatie op braakliggende rijstvelden in Suriname. De Surinaamse Landbouw 18: 47-63.

Dirven, J. G. P., 1970. Yield increase of tropical grassland by fertilization. Potassium symposium. 'Role of fertilization in the intensification of agricultural production'. Proceedings of the 9th Congress of the International Potash Institute, Antibes: $403-409$. 
Deinum, B. \& J. G. P. Dirven, 1971. Climate, nitrogen and grass. 4. The influence of age on chemical composition and in vitro digestibility of maize (Zea mays L.) and tall fescue (Festuca arundinacea Schreb.). Netherlands Journal of Agricultural Science 19: 264-272.

Dirven, J. G. P., 1971. De chemische samenstelling van enige grassoorten uit de gematigde en tropische gebieden, geteeld in Suriname. De Surinaamse Landbouw 19: 5-13.

Dirven, J. G. P. \& D. Oostendorp, 1971. Verslag van een studiereis naar Australië, Nieuw-Zeeland en Hawaiï. Gestencilde Mededeling No 24; Afdeling Landbouwplantenteelt en Graslandcultuur: 1-44.

Dirven, J. G. P., G. O. Nijland \& K. Wind, 1971. Aggregation of Trifolium repens at various fertilizations. Acta Botanica Neerlandica 20: 370-378.

Dovrat, A., J. G. P. Dirven \& B. Deinum, 1971. The influence of defoliation and nitrogen on the regrowth of Rhodes grass (Chloris gayana Kunth.). 1. Dry matter production and tillering. Netherlands Journal of Agricultural Science 19: 94101.

Deinum, B. \& J. G. P. Dirven, 1972. Climate, nitrogen and grass. 5. Influence of age, light intensity and temperature on the production and chemical composition of Congo grass (Brachiaria ruziziensis Germain et Evrard). Netherlands Journal of Agricultural Science 20: 125-132.

Dirven, J. G. P., 1972. Tropisch ruwvoer. Landbouwkundig Tijdschrift 84: 203207.

Dirven, J. G. P. \& F. W. v. Amson, 1972. Grasland op gepodzoliseerde gronden van het Lelydorplandschap. De Surinaamse Landbouw 20: 1-21.

Dovrat, A., B. Deinum \& J. G. P. Dirven, 1972. The influence of defoliation and nitrogen on the regrowth of Rhodes grass (Chloris gayana Kunth.). 2. Etiolated growth and non-structural carbohydrate, total-N and nitrate-N content. Netherlands Journal of Agricultural Science 20: 97-103.

Deinum, B. \& J. G. P. Dirven, 1973. Preliminary investigations on the digestibility of some tropical grasses grown under different temperature regimes. De Surinaamse Landbouw 21: 121-126.

Dirven, J. G. P., 1973. Tropical roughage. Thai Journal of Agricultural Science 6: 323-334.

Dirven, J. G. P., 1973. Tropical roughage. International course dairy cattle husbandry: 1-64.

Dirven, J. G. P. \& D. M. de Vries, 1973. Botanische Zusammensetzung von Pferdeweiden. Zeitschrift für Acker- und Pflanzenbau 137: 123-130.

Dirven, J. G. P., 1974. Exploitatie van tropische grasbestanden volgens oecologische principes. Post-Academiaal Onderwijs LH, 8 pp.

Minderhoud, J. W., P. F. J. van Burg, B. Deinum, J. G. P. Dirven \& M. L. 't Hart, 1974. Effects of high levels of nitrogen and adequate utilization on grassland productivity and cattle performance with special reference to permanent pastures in the temperate region. Proceedings 12 th International Grassland Congress (Moscow, 1974): 99-121.

Deinum, B. \& J. G. P. Dirven, 1975. Climate, nitrogen and grass. 6. Comparison of 
yield and chemical composition of some temperate and tropical grass species grown at different temperatures. Netherlands Journal of Agricultural Science 23: 69-82.

Dirven, J. G. P., 1975. Ruwvoedervoorziening van vee in de droge tijd. Verslag 15e Agromisaweek 1975.

Dirven, J. G. P. \& J. H. Neuteboom, 1975. Bemesting en plantkundige samenstelling van grasland. Stikstof $80: 224-231$.

Deinum, B. \& J. G. P. Dirven, 1976. Climate, nitrogen and grass. 7. Comparison of production and chemical composition of Brachiaria ruziziensis and Setaria sphacelata grown at different temperatures. Netherlands Journal of Agricultural Science 24: 67-78.

Dirven, J. G. P., 1977. Graslandproblemen in Cuba. Gebundelde verslagen NVWV 18: 7-16.

Dirven, J. G. P., 1977. Beef and milk production from cultivated tropical pastures. A comparison with temperate pastures. Stikstof (Dutch nitrogen fertilization review) 20: 2-15.

Dirven, J. G. P. \& B. Deinum, 1977. The effect of temperature on the digestibility of grasses. An analysis. Forage Research 3: 1-17.

Dirven, J. G. P., L. J. M. v. Soest \& K. Wind, 1979. The influence of photoperiod on head formation in some Brachiaria species and Chloris gayana vc. Masaba. Netherlands Journal of Agricultural Science 27: 48-60.

Dirven, J. G. P., 1980. Tropical grassland management based on ecological principles. Vakgroep Landbouwplantenteelt en Graslandkunde, Mededeling No 53; $8 \mathrm{pp}$.

Dirven, J. G. P. \& K. Wind, 1980. Zodedichtheid van grasvegetaties. III. Spruitdichtheid van grasbestanden en toepassing van de telmethode. Vakgroep Landbouwplantenteelt, Grasland- en Onkruidkunde, Mededeling No 51; $17 \mathrm{pp}$.

Dirven, J. G. P. \& K. Wind, 1981. De invloed van natrium- en kalibemesting op de opbrengst en gehalten aan natrium en kalium bij verschillende herkomsten van Festuca rubra L. Vakgroep Landbouwplantenteelt en Graslandkunde, Mededeling No 60;16 pp.

Dirven, J. G. P. \& I. G. H. Dulder, 1982. Graasgewoonten van rundvee in de huidige tropen (Suriname). Vakgroep Landbouwplantenteelt en Graslandkunde, Mededeling No 70; $34 \mathrm{pp}$.

Dirven, J. G. P. \& K. Wind, 1982. De invloed van bemesting op de beworteling van verschillende grassoorten en -rassen. Vakgroep Landbouwplantenteelt en Graslandkunde, Mededeling No 61; 33 pp.

Dirven, J. G. P. \& K. Wind, 1982. Intensivering van de graslandexploitatie en de plantkundige samenstelling van de grasmat. Vakgroep Landbouwplantenteelt en Graslandkunde, Mededeling No 65;24 pp.

Dirven, J. G. P., K. Wind \& E. H. H. Wolbers, 1982. De spruit/wortelverhouding bij buntgras (Corynephorus canescens (L.) P.B.). Vakgroep Landbouwplantenteelt en Graslandkunde, Mededeling No 67; 17 pp.

Dirven, J. G. P., 1983. Loekoentoegras (Ischaemum timorense Kunth.) in Suriname. Een evaluatie. Vakgroep Landbouwplantenteelt en Graslandkunde, Me- 
dedeling No 72; $29 \mathrm{pp}$.

Dirven, J. G. P., 1983. De overgangshooilanden Anthoxanthum odoratum - Holcus lanatus - Rumex acetosa. Vakgroep Landbouwplantenteelt en Graslandkunde, Mededeling No 75; $20 \mathrm{pp}$.

Dirven, J. G. P., 1983. Bamboegras- en zuurgrasvegetaties op braakliggende rijstvelden in Suriname. Vakgroep Landbouwplantenteelt en Graslandkunde, Mededeling No 76; $17 \mathrm{pp}$.

Elberse, W. Th., J. P. van den Bergh \& J. G. P. Dirven, 1983. The effect of use and mineral supply on the botanical composition and yield of old grassland on heavyclay soil. Netherlands Journal of Agricultural Science 31: 63-88.

Dirven, J. G. P., 1984. De minerale samenstelling van afzonderlijke graslandplanten. Vakgroep Landbouwplantenteelt en Graslandkunde, Mededeling No 81; $14 \mathrm{pp}$.

Dirven, J. G. P., K. Wind \& W. Pik, 1984. De invloed van de daglengte en temperatuur op de bloeihalmvorming van 4 cultivars van Setaria sphacelata (Schumach) Stapf and Hubbard ex Moss (Syn. Setaria anceps Stapf). Vakgroep Landbouwplantenteelt en Graslandkunde, Mededeling No 80; 12 pp. 